\title{
SENAI CIMATEC in the Scnario of Coronavirus Pandemic
}

\author{
Leone Peter Andrade ${ }^{1 *}$, Roberto Badaró ${ }^{2}$ \\ ${ }^{1}$ Editor-in-Chief and Director of Integrated Campus of Manufacturing and Technology (CIMATEC); ${ }^{2}$ Deputy Editor and \\ Medical Director of ISI (SENAI Institute of Innovation in Advanced Health Systems); Salvador, Bahia, Brazil
}

Historically, every major change is preceded by events that cause large social and economic disruption. The new coronavirus pandemic has posed challenges to the health system, people, and the economy, leading to abrupt social changes. There was a change in the entire world scenario and people's lives were affected in many ways: health care, social behavior and, mainly, in how to establish the parameters of a new economy.

According to a survey by the National Confederation of Industry (CNI), $79 \%$ of industries claim to have suffered a reduction in orders. About $53 \%$ say the fall was intense. The data showed $86 \%$ of the companies are struggling to receive inputs and $83 \%$ face problems in transport logistics, both for products and raw materials.

This scenario leads to a great rush to adapting the new social behavior and economic models. New investments in the economic and governmental sector and behavioral and social changes that would happen in years are occurring in a few months, thanks to the tools of industry 4.0.

Industry 4.0 is a concept recently proposed and encompasses the main technological innovations in the fields of automation, control, and information technology applied to manufacture processes. The term appeared in Germany, in the 2011 edition of the Hannover Fair, and proposes to cross the boundaries between the digital, the physical and the biological world.

Thus, although the negative economic impact has been huge since the pandemic was declared by the World Health Organization in March 2020, new achievements are being made by industry 4.0 focused on health, since this impact has caused the world economy to shrink, generating unemployment and social instability.

Developed countries, such as the United States of America, Germany, and Japan, and the main emerging countries, such as China and India, have outlined strategies to strengthen the industrial sector and make industry 4.0 a reality at this time. However, the spread of SARS-COV-2, the virus that caused the COVID-19 pandemic, revealed a bleak reality: most countries were not prepared to face a large-scale health emergency. Also, hospitals are not equipped with a sufficient number of ventilators and respirators, essential equipments to save lives in the most serious cases of COVID-19, and the domestic industrial parks did not have the necessary installed capacity to meet rapidly expanding demand. The wide majority of countries faced their high dependence on the import of these materials or supplies from China, such as personal protective equipment (PPE) for health teams, face shields, gloves, masks surgical, disinfectants, hydroalcoholic gel and masks for the general population. Another bottleneck that has been noted since the beginning of the pandemic was the insufficiency of diagnostic test kits for SARS-COV-2. Thus, given the difficulty in obtaining medical and hospital equipment and materials, which are fundamental for the management of the symptoms of COVID-19, the conversion of industrial production lines has helped many countries to meet the strong increase in demand of many products previously imported, mainly about the manufacture of ventilators, mechanical respirators, and personal protective equipment (PPE).

Received on 2 March 2020; revised 9 June 2020.Address for correspondence: Dr. Roberto Badaró. Centro Universitário SENAI CIMATEC. Av. Orlando Gomes, 1845, Piatã. Zip Code: 41650-010. Salvador, Bahia, Brazil. E-mail: jbth@jbth.com.br.

J Bioeng. Tech. Appl. Health 2020;3(1):1-2. (02020 by SENAI CIMATEC. All rights reserved. 
Figure 1. Campus of SENAI CIMATEC.

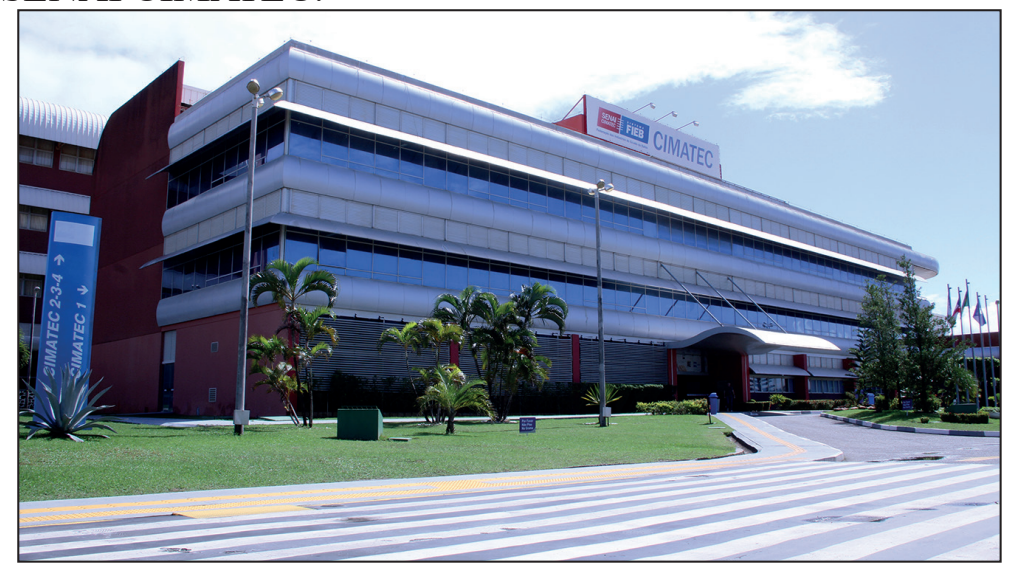

However, despite the negative economic aspect and the need to innovate to reconverting production lines, the industries began to put into practice the knowledge concerning industry 4.0, since Fourth Industrial Revolution gave us the tools we need to battle this global threat (artificial intelligence, Big Data, neural network, IoT). The new technologies have the capability of providing better digital solutions for our daily lives during this crisis. Several benefits help us for mitigating effects of COVID-19 pandemic and searching treatments and vaccines, as follow:

- Providing medical part in time using smart supply chain.

- Using robotic based treatment of the infected patient to reduce medical risks.

- Using virtual reality for training purpose.

- Promoting a flexible working environment of treatment.

- Digital Technologies can help people to perform daily life work during the lockdown.

- Expansion use of the Telemedicine.

- Researchers can employ these technologies for social and media platforms to identify unusual information.

- The creation of resourced databases to track and predict infectious risk.

- Artificial intelligence (AI) could be used to enabling states to manage the caseload.

- AI can be a valuable triage tool through virtual chatbots, a considerably important resource in scenarios of high clinical demand.
- Using of AI to new image methods for COVID-19.

- The power of robots and drones, which have proven instrumental in reducing interpersonal contact by facilitating the delivery of food and medication and the disinfection of public spaces.

- Implementing universal use of masks in conjunction with lockdown efforts, successfully driving a flattening of the curve.

- Implementing curfews to mitigate community transmission and have communicated rapidly, transparently, and thoroughly.

The Fourth Industrial Revolution has equipped society with highly potent tools and we must harness their capabilities, where possible, to win this fight. So, COVID-19 accelerates this process and sentences our definitive entry to the fourth Industrial Revolution.

In this sense, aligned with industry 4.0, SENAI CIMATEC (Figure 1) established a proactive position in the face of the COVID-19 pandemic, promoting research, implementing plans, activities, and actions that contemplate both the redirection of the sector's industries to the health area, as well as the new technologies of industry 4.0. The position of SENAI CIMATEC reaffirms its commitment to society at decisive moments and ensures its efforts in health care against the pandemic. 